\title{
Previsão do preço do café arábica: uma aplicação de redes neurais CNN-BLSTM
}

\author{
Arabica coffee price forecast: a neural network application CNN-BLSTM \\ Predicción del precio del café arábica: una aplicación de red neuronal CNN-BLSTM
}

Recebido: 24/01/2022 | Revisado: 01/02/2022 | Aceito: 05/02/2022 | Publicado: 10/02/2022

\author{
José Airton Azevedo dos Santos \\ ORCID: https://orcid.org/0000-0002-2568-5734 \\ Universidade Tecnológica Federal do Paraná, Brasil \\ E-mail: airton@utfpr.edu.br
}

\begin{abstract}
Resumo
Este trabalho propõe a utilização da rede neural CNN-BLSTM como ferramenta de previsão do preço do café arábica. A base de dados disponibilizada pelo CEPEA (Centro de Estudos Avançados em Economia Aplicada) apresenta uma série histórica, do preço do café arábica, no período entre janeiro de 1997 e dezembro de 2021. Modelos de previsão baseados em redes neurais LSTM, BLSTM, CNN e CNN-BLSTM foram implementados, na linguagem Python, utilizando o framework Keras. Resultados obtidos, dos quatro modelos, foram comparados por meio das métricas MAE, RMSE e MAPE. Verificou-se, para um horizonte de 6 meses, que o modelo CNN-BLSTM apresentou melhor desempenho.
\end{abstract}

Palavras-chave: Redes neurais artificiais; Café arábica; Keras; Python.

\begin{abstract}
This work proposes the use of the CNN-BLSTM neural network as a tool to predict the price of arabica coffee. The database provided by CEPEA (Center for Advanced Studies in Applied Economics) presents a historical series of the price of arabica coffee, in the period between January 1997 and December 2021. Forecast models based on neural networks LSTM, BLSTM, CNN and CNN-BLSTM were implemented, in the Python language, using the Keras framework. Results obtained, from the four models, were compared using MAE, RMSE and MAPE metrics. It was verified, for a horizon of 6 months, that the CNN-BLSTM model presented better performance.
\end{abstract}

Keywords: Artificial neural networks; Arabica coffee; Keras; Python.

\section{Resumen}

Este trabajo propone el uso de la red neuronal CNN-BLSTM como herramienta para predecir el precio del café arábica. La base de datos proporcionada por CEPEA (Centro de Estudios Avanzados en Economía Aplicada) presenta una serie histórica del precio del café arábica, en el período comprendido entre enero de 1997 y diciembre de 2021. Modelos de pronóstico basados en redes neuronales LSTM, BLSTM, CNN y CNN-BLSTM fueron implementados, en lenguaje Python, usando el framework Keras. Los resultados obtenidos, de los cuatro modelos, se compararon utilizando métricas MAE, RMSE y MAPE. Se verificó, para un horizonte de 6 meses, que el modelo CNN-BLSTM presentó mejor desempeño.

Palabras clave: Redes neuronales artificiales; Café arábica; Keras; Python.

\section{Introdução}

O café, com grande participação econômica no Brasil, possui uma importância inegável no cenário mundial. Sua importância pode ser constatada por meio do consumo interno, participação na pauta de exportações e capacidade de geração de empregos. O café é um dos produtos mais comercializados, ficando, em valor de mercado, apenas atrás do petróleo. Seu cultivo, processamento, comercialização e transporte proporcionam milhões de empregos em todo o mundo (Tagiba et al., 2010; Auriglietti et al., 2021; Barros, 2021; SINDICAFÉ-MG, 2022; Faria et al., 2004).

As espécies Coffea Canephora (Café Conilon) e Coffea Arabica (Café Arábica) dominam o mercado brasileiro. Sendo que a espécie arábica, em 2019, representou cerca de 70\% da safra nacional (aproximadamente 35 milhões de sacas de $60 \mathrm{~kg}$ ), o conilon, também chamado de robusta, ficou com 30\% (cerca de 15 milhões de sacas de 60kg) (Martins, 2021). 
Os produtores de café, assim como os produtores de outros grãos, estão sujeitos a volatilidade dos preços. Portanto, a previsão, de preços futuros do café, torna-se um fator importante para esta atividade produtiva. Possibilitando aos produtores, maior segurança em seus planejamentos e negociações (Santos, 2021; Marujo, 2021; Auriglietti et al., 2021).

Dentre as técnicas que podem ser utilizadas, para previsão do preço do café, estão as Redes Neurais Artificiais (RNAs). As redes neurais, sistemas computacionais que imitam a maneira como o cérebro humano funciona, são ferramentas de grande importância em um sistema de planejamento. Podem ser consideradas como um esquema de processamento capaz de armazenar conhecimento e disponibilizá-lo para determinada aplicação (Pinheiro et al., 2020; Bastiani et al., 2018; Haykin, 2005; Santos et al., 2020).

Diversos trabalhos, devido à importância do assunto, utilizaram métodos de previsão de séries temporais na análise do preço do café. Silva (2018) analisou, por meio da metodologia ARIMA (Autorregressivo Integrado de Médias Móveis), o comportamento dos preços médios, recebidos pelo produtor de janeiro de 2005 a dezembro de 2016, do café arábica. Concluiu que a modelagem ARIMA apresenta um adequado poder de previsão de curto prazo. Faria (2011) também analisou, no período entre maio de 2008 e setembro de 2009, o mercado futuro do café arábica por meio de modelos ARIMA. Ayyanathan \& Kannammal (2014) analisaram, por meio de Redes Neurais Artificiais e Máquinas de Vetor de Suporte, o preço de ações, no mercado indiano, de uma empresa líder na exportação de café. Novanda et al. (2018) analisaram e comparam modelos, ARIMA e de Médias Móveis, para prever, no mercado doméstico e mundial, a volatilidade do preço do café. Concluíram, neste trabalho, que os modelos ARIMA se mostraram mais adequados para previsão do preço do café. Lopes (2018) estudou modelos, conhecidos como Statistical Machine Learning, para previsão do preço do café brasileiro. Conclui que o modelo de Máquina de vetor de Suporte, com kernel linear, obteve melhor acurácia na previsão do preço do café. Já Deina et al. (2021) realizaram, para o período entre setembro de 1996 e dezembro de 2018, a previsão dos preços dos cafés arábica e robusta. Como preditores utilizaram os seguintes modelos: Suavização Exponencial (ES), Autoregressivo (AR), Autorregressivo Integrado de Médias Móveis (ARIMA), Perceptron Multicamadas (MLP) e Máquinas de Aprendizado Extremo (ELM). Concluíram que o modelo ELM apresentou melhor desempenho na previsão do preço do café.

Apesar da importância do café, na economia brasileira, existem poucos trabalhos que utilizam redes neurais híbridas, como a CNN-BLSTM, na previsão do preço do café. Geralmente, são utilizados modelos tradicionais como os modelos ARIMA, SVM, MLP, etc (Lu et al., 2020).

Neste contexto, este trabalho propõe a utilização de redes CNN-BLSTM (Convolutional Neural Network Bidirectional Long Short-Term Memory) para previsão do preço do café arábica. Este método híbrido utiliza a rede CNN para extrair os recursos dos dados de entrada e a rede BLSTM para prever o preço do café.

Este trabalho está organizado da seguinte maneira. Na Seção 2 são descritas as metodologias empregadas na previsão do preço do café arábica. Na Seção 3 são apresentados os resultados obtidos da aplicação dos modelos, de Redes Neurais Artificiais, em observações mensais do preço do café arábica. Comentários finais e conclusões finalizam o trabalho.

\section{Metodologia}

Nesta seção aborda-se a metodologia utilizada, para realizar essa pesquisa, detalhando os métodos propostos para realização dos experimentos, bem como as técnicas utilizadas.

\subsection{Redes LSTMs (Long Short-Term Memory)}

As redes neurais LSTMs, propostas por Graves et al., (1977), são muito utilizadas em tarefas que envolvam entradas sequênciais, como séries temporais, reconhecimento de dígitos, reconhecimento de voz, tradução automática, etc (Gazzola, 
2017). Estas redes demostraram excelente habilidade, em relação a outras redes neurais, para apreender dependências de longo prazo (Santos et al., 2021).

A topologia, de um neurônio de uma LSTM, é baseada em uma célula de memória (Figura 1). A célula de memória, para prever estados futuros, possui portas que tem a capacidade de adicionar, descartar ou atualizar informações no tempo. Na célula LSTM tem-se três portas:

- Porta de Esquecimento $\left(f_{t}\right)$ : determina quais informações devem ser descartadas pela célula.

- Porta de Entrada $\left(i_{t}\right)$ : determina os valores de entrada para atualizar o estado da célula $\left(C_{t}\right)$.

- Porta de Saída $\left(O_{t}\right)$ : determina o que produzir com base na entrada e na memória da célula.

Figura 1. Célula LSTM.

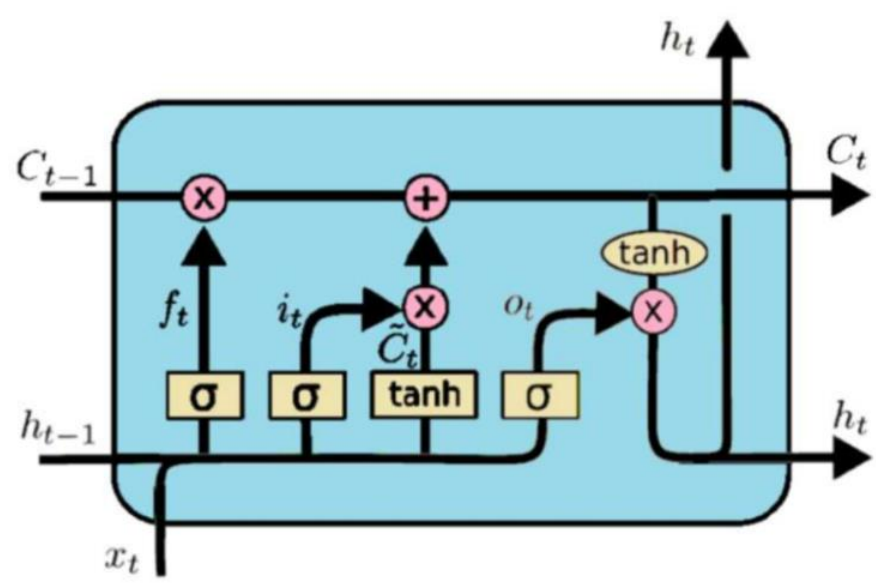

Fonte: Graves et al., (2014).

As equações, apresentadas a seguir, descrevem uma célula LSTM (Graves \& Jaitly, 2014; Santos \& Spancerski, 2021).

$$
\begin{gathered}
f_{\mathrm{t}}=\sigma_{g}\left(W_{f} x_{\mathrm{t}}+U_{f} h_{\mathrm{t}-1}+b_{f}\right) \\
\tilde{i}_{\mathrm{t}}=\sigma_{g}\left(W_{\mathrm{i}} x_{\mathrm{t}}+U_{\mathrm{i}} h_{\mathrm{t}-1}+b_{\mathrm{i}}\right) \\
o_{\mathrm{t}}=\sigma_{g}\left(W_{e} x_{\mathrm{t}}+U_{e} h_{\mathrm{t}-1}+b_{\mathrm{e}}\right) \\
c_{\mathrm{t}}=f_{\mathrm{t}} * c_{\mathrm{t}-1}+\tilde{i}_{\mathrm{t}} * \sigma_{\mathrm{c}}\left(W_{c} x_{\mathrm{t}}+U_{c} h_{\mathrm{t}-1}+b_{c}\right) \\
h_{\mathrm{t}}=o_{\mathrm{t}} * \sigma_{h}\left(c_{\mathrm{t}}\right)
\end{gathered}
$$

Onde: $x_{t}$ - sequência de entrada, $C_{t}$ - estado da célula. $\sigma_{g}-$ Função sigmoide, $\sigma_{h^{-}}$- Função tangente hiperbólica, W e U- Matrizes de peso, b- bias e $h_{t^{-}}$é o bloco de saída para o tempo $t$. 


\subsection{Redes LSTM Bidirecional (BLSTMs)}

As redes Bidirecionais BLSTMs, introduzidas por Schuster e Paliwal em 1997, são uma extensão dos modelos LSTMs. São muito utilizadas no reconhecimento de voz e classificação de texto, mas muito pouco aplicadas em previsões de séries temporais (Althelaya, 2018).

As redes BLSTMs processam, por meio de duas camadas LSTM, a entrada em duas direções. Tem-se uma sequência forward $\left(\vec{h}_{\mathfrak{t}}\right)$ e uma sequência backward $\left(\vec{h}_{\mathfrak{t}}\right)$ (Figura 2). As camadas LSTMs são conectadas a camada de saída. Isto faz com que este tipo de rede possa compreender melhor o contexto (Graves et al., 2014; Nelson et al., 2017; Zao et al., 2017; Santos et al., 2021).

Figura 2. Rede BLSTM.

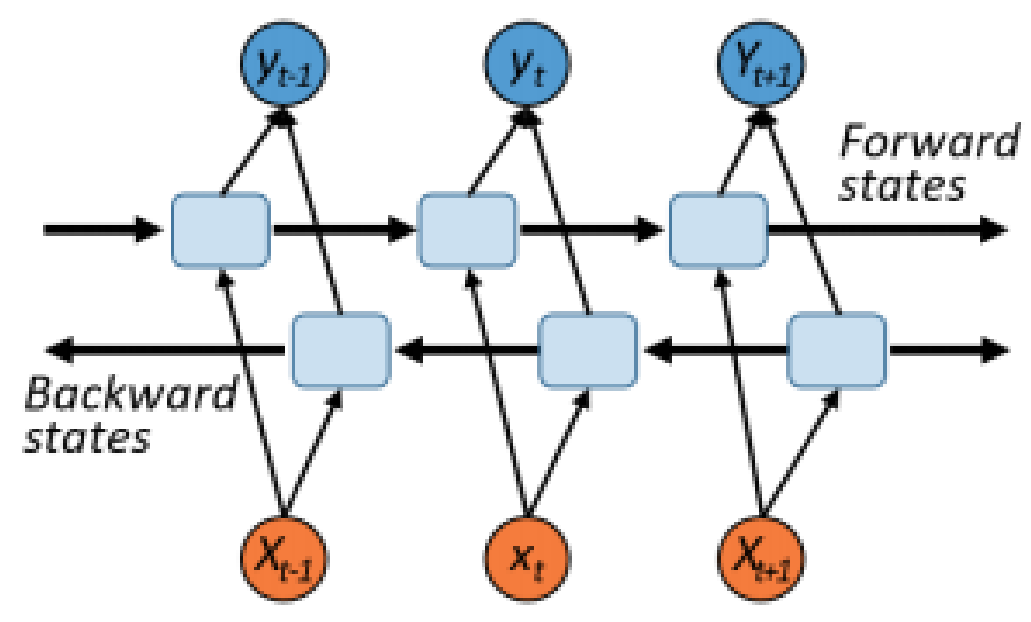

Fonte: Sun et al., (2018).

As sequências $\vec{h}_{t}$ e $\breve{h}_{t}$ são combinadas para encontrar a sequência de saída y (Fazard \& Gulliver, 2019; Mousa \& Schuller, 2016):

$$
\begin{gathered}
\vec{h}_{\mathrm{t}}=\sigma\left(W x_{\mathrm{t}}+W \vec{h}_{\mathrm{t}-1}+b\right) \\
\hat{h}_{\mathrm{t}}=\sigma\left(W x_{\mathrm{t}}+W \hat{h}_{\mathrm{t}-1}+b\right) \\
y_{t}=W \vec{h}_{\mathrm{t}}+W \tilde{h}_{\mathrm{t}}+b
\end{gathered}
$$

Onde: $W$ - Matriz de pesos, $b$ - bias e $\sigma$ - função de ativação.

\subsection{Redes CNN-BLSTM}

As redes CNNs são utilizadas, com muito sucesso, em classificação de imagens. Sua versão 1D é aplicada para tratar sequências de dados, tais como séries temporais, gravações de áudio e processamento de linguagem natural (Lawal, 2021).

Uma rede CNN é composta por uma sequência de camadas. Existem, além da camada de entrada, três camadas principais: camada convolucional, camada de pooling e uma camada totalmente conectada (Figura 3) (Pacheco, 2016). 
Figura 3. Estrutura da rede CNN.

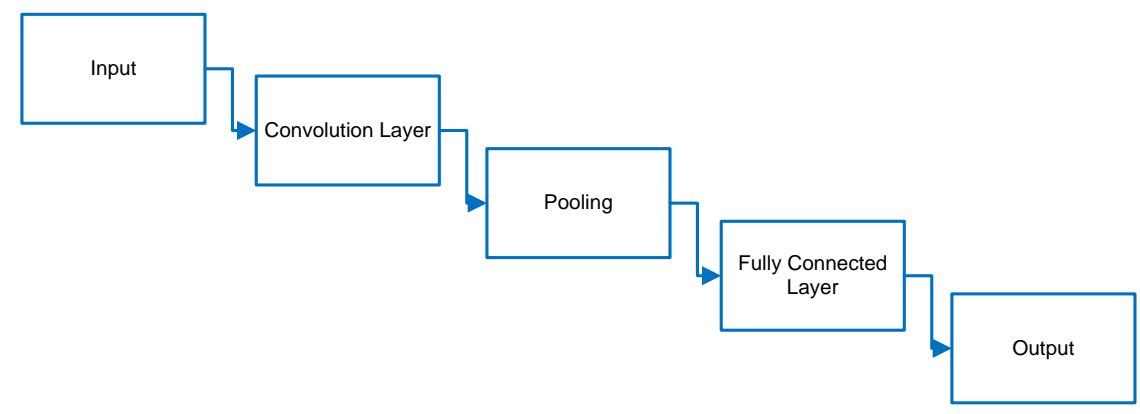

Fonte: Autores.

O modelo híbrido CNN-BLSTM é uma arquitetura, baseada na rede BLSTM, projetada para lidar com previsões de sequências. Envolve o uso da rede CNN, como camada inicial, para a extração de características a partir dos dados de entrada. O resultado da rede CNN, o vetor FM, é repassado a rede BLSTM.

$$
F M=\operatorname{CNN}\left(x_{\mathrm{t}}\right)
$$

Onde $x_{t}$ é o vetor de entrada.

\subsection{Base de dados e etapas do trabalho}

Para previsão do preço do café arábica, em US\$ por saca de $60 \mathrm{~kg}$ (US\$/60kg), utilizou-se uma base de dados com 300 meses (Jan/1997 - Dez/2021) disponibilizada pelo CEPEA (Centro de Estudos Avançados em Economia Aplicada) (CEPEA, 2022). A série histórica, disponibilizada pelo CEPEA, é apresentada na Figura 4.

Figura 4. Gráfico ilustrativo da série temporal do preço do café arábica.

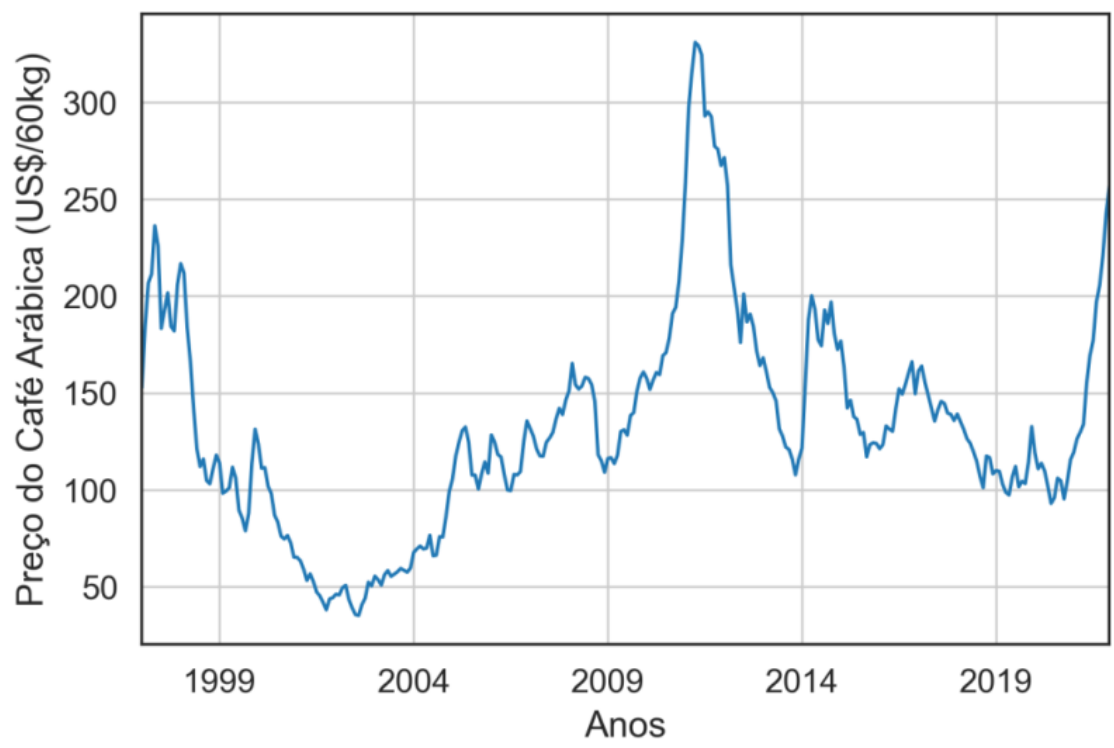

Fonte: Autores.

Este trabalho foi dividido em três etapas: 
Etapa 1 (Coleta e análise de dados): Inicialmente, na primeira etapa, realizou-se uma análise exploratória dos dados obtidos do CEPEA. Na sequência, a base de dados, do preço do café arábica, foi dividida em três conjuntos de dados: O conjunto de treinamento com os 205 primeiros dados, o conjunto de validação formado pelos seguintes 89 dados e o conjunto de teste formado pelos 6 dados restantes.

Os dados, para evitar que o desempenho dos algoritmos fossem afetados, passaram por uma transformação logarítmica, seguida por uma normalização. Neste trabalho, a normalização dos dados, para o intervalo [0,1], foi realizada por meio da função MinMaxScaler() da biblioteca Scikit-learn.

Etapa 2 (Treinamento e Validação): Na segunda etapa, treinamento e validação, foi selecionado, por meio das métricas MAE, RMSE e MAPE, os melhores modelos LSTM, BLSTM, CNN e CNN-BLSTM.

Etapa 3 (Teste): Na última etapa, etapa de teste, testaram-se os modelos para dados que não participaram da etapa de treinamento e validação (julho, agosto, setembro, outubro, novembro e dezembro de 2021).

\subsection{Métricas}

Neste trabalho, os modelos foram avaliados pelas métricas, MAE (Erro Médio Absoluto), RMSE (Raiz Quadrada do Erro Médio Quadrático) e MAPE (Erro Médio Absoluto Percentual), apresentadas na Tabela 1 (Cankurt et al., 2015; Pinheiro et al., 2020; Bastiani et al., 2018). O MAE mede a média da diferença entre o valor real e o valor predito. O RMSE, raiz do erro médio quadrático da diferença entre a predição e o valor real, dá um peso maior para desvios grandes (porque são elevados ao quadrado). Já o MAPE mede o tamanho do erro em termos percentuais.

Tabela 1. Equações: RMSE, MAE e MAPE.

\begin{tabular}{ccc}
\hline Métrica & Equação & Valor Perfeito \\
\hline MAE & $\frac{1}{n} \sum_{1}^{n}\left|\left(y_{i}-\hat{y}_{i}\right)\right|$ & MAE $=0$ \\
RMSE & $\sqrt{\frac{1}{n} \sum_{i=1}^{n}\left(\left(y_{i}-\hat{y}_{i}\right)^{2}\right)}$ & RMSE $=0$ \\
MAPE & $\frac{1}{n} \sum_{i=1}^{n}\left|\left(y_{i}-\hat{y}_{i}\right) / y_{i}\right| \times 100$ & MAPE $=0$ \\
& \multicolumn{2}{c}{ Fonte: Cankurt et al., (2015). }
\end{tabular}

Onde: $y_{i}$ é o valor real do período $i, \widehat{y}_{i}$ é a previsão para o período $i$ e $n$ é o número de observações.

\section{Resultados e Discussão}

Inicialmente, neste trabalho, realizou-se uma análise descritiva dos dados fornecidos pelo CEPEA (Tabela 2).

Tabela 2. Análise descritiva do preço do café arábica. 


\begin{tabular}{cc}
\hline Resumo Descritivo & Preço \\
\hline Quantidade & 300 \\
\hline Média (US\$/60kg) & 132,86 \\
\hline Mínimo (US\$/60kg) & 35,21 \\
\hline Máximo (US\$/60kg) & 330,82 \\
\hline Desvio Padrão (US\$/60kg) & 56,73 \\
\hline Coeficiente de Variação $(\%)$ & 42,69 \\
\hline \multicolumn{2}{c}{ Fonte: Autores. }
\end{tabular}

Pode-se observar, dos dados apresentados na Tabela 2, que o preço ficou, para o período em estudo, em média de 132,86 US\$/60kg. Apresentando, neste período, preços mínimo e máximo de 35,21 US\$/60kg e 330,82 US\$/60kg, respectivamente. Observa-se também, da Tabela 2, um coeficiente de variação de 42,69\%, considerado alto, indicando variabilidade dos dados.

\subsection{Treinamento e validação}

Vários modelos de redes neurais LSTM, BLSTM, CNN e CNN-BLSTM foram testados, na previsão do preço do café arábica, utilizando como indicador de desempenho as métricas MAE, RMSE e MAPE. As redes neurais foram treinadas com 205 amostras (70\%) e validadas com 89 amostras (30\%). Na Tabela 3 apresentam-se os valores dos parâmetros utilizados pela rede neural CNN-BLSTM.

Tabela 3. Parâmetros da rede CNN-BLSTM.

\begin{tabular}{lc}
\hline \multicolumn{2}{c}{ Parâmetros } \\
\hline Convolution layers filters & 8 \\
\hline Convolution layer Kernel_size & 5 \\
\hline Convolution layer activation function & relu \\
\hline Convolution layer padding & Same \\
\hline Pooling layer pool_size & 1 \\
\hline Pooling layer padding & Same \\
\hline Number of hidden units in LSTM layer & 8 \\
\hline LSTM layer activation function & relu \\
\hline Time_step & 24 \\
\hline Batch_size & 50 \\
\hline Learning rate & 0,001 \\
\hline Optmizer & Adam \\
\hline Loss function & MSE \\
\hline Epochs & 144 \\
\hline
\end{tabular}

Na Figura 5 apresentam-se os resultados da predição, de treinamento e validação, para o modelo CNN-BLSTM. Pode notar, por meio da figura, a boa aderência dos dados previstos com os dados reais.

Figura 5. Previsão de treino e validação - CNN-BLSTM. 


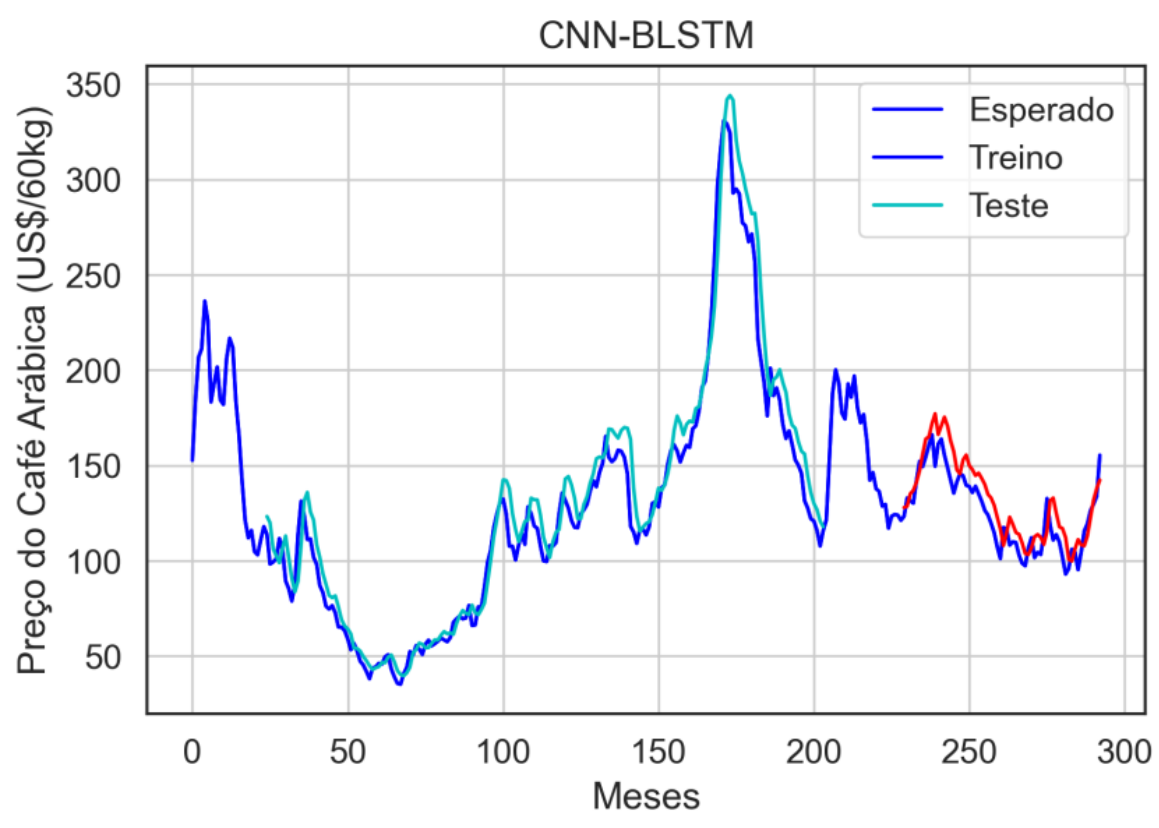

Fonte: Autores.

\subsection{Teste}

Nas Tabelas 4 e 5 apresentam-se os dados, observados (CEPEA), preditos (LSTM, BLSTM, CNN e CNN-BLSTM) e os valores das métricas MAE, RMSE e MAPE, para os seis meses que não participaram da etapa de treinamento e validação (Conjunto de teste).

Tabela 4. Dados observados e preditos (US\$/60kg).

\begin{tabular}{cccccc}
\hline Mês & CEPEA & LSTM & BLSTM & CNN & CNN-BLSTM \\
\hline jul/21 & 177,1491 & 178,5685 & 189,8003 & 176,52846 & 179,85732 \\
\hline ago/21 & 196,9605 & 190,2866 & 211,379 & 188,78805 & 193,3991 \\
\hline set/21 & 205,4776 & 198,0594 & 228,745 & 200,08595 & 204,27512 \\
\hline out/21 & 220,7775 & 217,305 & 258,9777 & 214,84001 & 225,46373 \\
\hline nov/21 & 242,4 & 240,4327 & 303,505 & 228,52963 & 249,16566 \\
\hline dez/21 & 256,6686 & 251,7204 & 344,2541 & 242,13351 & 258,7597 \\
\hline
\end{tabular}

Fonte: Autores.

Tabela 5. Métricas - Conjunto de teste.

\begin{tabular}{cccc} 
& \multicolumn{3}{c}{ Métricas } \\
\cline { 2 - 4 } Modelos & MAE & RMSE & MAPE (\%) \\
\hline LSTM & 4,31 & 4,86 & 2,02 \\
\hline BLSTM & 39,54 & 47,91 & 17,07 \\
\hline CNN & 7,82 & 9,69 & 6,47 \\
\hline CNN-BLSTM & 1,58 & 1,89 & 0,68 \\
\hline & Fonte: Autores.
\end{tabular}

Nota-se, por meio dos resultados apresentados na Tabela 5, que o modelo CNN-BLSTM apresenta erros bem menores que os modelos LSTM, BLSTM e CNN. Observa-se também, ao comparar a rede LSTM com a CNN-BLSTM, um decréscimo 
dos valores das métricas MAE, RMSE e MAPE, da rede CNN-BLSTM em relação a rede LSTM, de 63,34\%, 61,11\% e $66,34 \%$, respectivamente. O que corrobora com o bom ajuste, dos valores preditos com os valores reais, apresentado na Figura 6.

Figura 6. Preço do café - conjunto de teste.

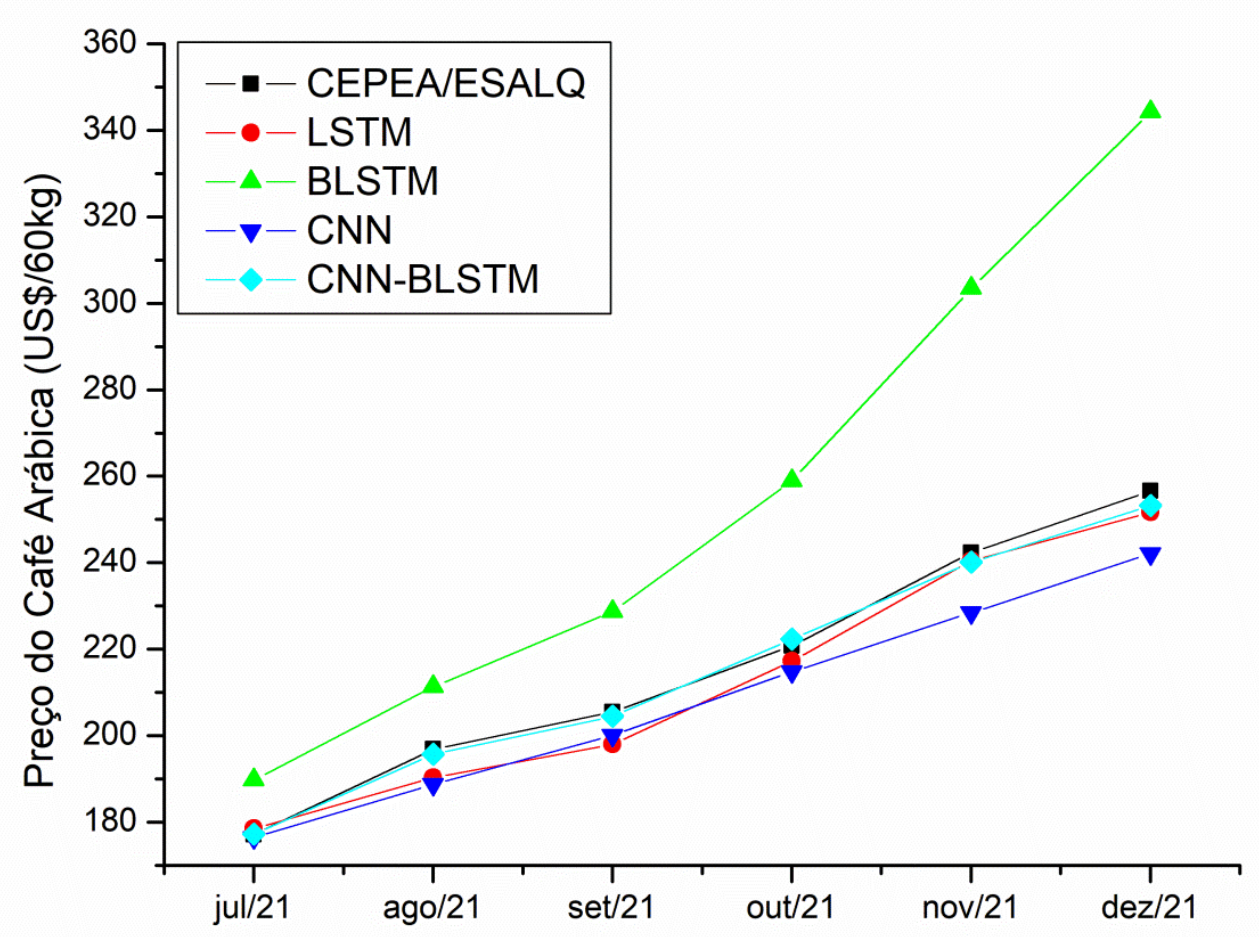

Fonte: Autores.

\section{Considerações Finais}

Buscou-se, neste estudo, determinar a viabilidade da utilização do modelo CNN-BLSTM para previsão de preços futuros do café arábica. Para isto, foi utilizado dados da série histórica, do preço do café arábica, no período entre janeiro de 1997 e dezembro de 2021. Os modelos LSTM, BLSTM, CNN e CNN-BLSTM, construído com a base de dados disponibilizada pelo CEPEA, passaram pelas etapas de validação e teste.

Inicialmente, na etapa de treinamento e validação, validaram-se os modelos LSTM, BLSTM, CNN e CNN-BLSTM. Na sequência, na etapa de teste, utilizando os parâmetros encontrados para os modelos na etapa de treinamento e validação, estimaram-se os valores, do preço do café arábica, de julho de 2021 a dezembro de 2021 (conjunto de teste). Os dados obtidos, pelos quatro modelos, foram comparados com valores reais. O modelo com melhor desempenho no conjunto de teste, considerando as métricas MAE, RMSE e MAPE, foi o CNN-BLSTM.

Conclui-se, por meio dos resultados obtidos, que a rede híbrida CNN-BLSTM pode ser utilizada como ferramenta de gestão, por produtores e compradores desta commodity, nos processos de tomada de decisão e planejamento futuro.

\section{Referências}


Research, Society and Development, v. 11, n. 3, e3511326101, 2022

(CC BY 4.0) | ISSN 2525-3409 | DOI: http://dx.doi.org/10.33448/rsd-v11i3.26101

Aurigliett, L. M. M. \& Tonin, J. M. (2021). Relação entre base, volatilidade e liquidez: evidências para o mercado futuro de café no Brasil. Revista AE\&S, 2(1), 1-4. https://doi.org/10.22167/2675-20210003.

Althelaya, K. A. (2018). Evaluation of bidirectional LSTM for short and long term stock market prediction. In: International Conference on Information as Communication System (ICICS), Irbid, Jordan.

Ayyanathan, N. \& Kannammal, A. (2014). Share price time series forecasting for effective supply chain information Exchange. International Journal of Logistics Systems and Management, 18(1). https://doi.org/10.1504/IJLSM.2014.062125.

Barros, D. (2021). Dados sobre Café no Brasil: Consumo, Produção e Exportação. Disponível em: https://reviewcafe.com.br/dicas-e-receitas/dados-sobrecafe-no-brasil/. Acesso em: 15 jan. 2022.

Bastiani, M., Santos, J. A. A., Schmidt, C. A P. \& Sepulveda, G. P. L. (2018). Application of data mining algorithms in the management of the broiler production. Geintec. 8(4), 4574-4587. https://doi.org/10.7198/geintec.v8i4.1275.

Cankurt, S. \& Subasi, A. (2015). Comparasion of linear regression and neural network models forecasting tourist arrivals to turkey. Eurasian Journal of Science \&Engineering.

CEPEA (2022). Indicador do café arábica. https://www.cepea.esalq.usp.br/br/indicador/cafe.aspx.

Deina, C. et al. (2021). A methodology for coffee price forecasting based on extreme learning machines. Information Processing in Agriculture. https://doi.org/10.1016/j.inpa.2021.07.003.

Faria, T. A. (2011). Mercado future do café: um estudo de caso. Revista de Estudos Sociais, 13(26), 138-156.

Faria, A. C. S. \& Manolescu, F. M. K. (2004) A produção de café no Brasil. <http://www.inicepg.univap.br/cd/INIC_2004/trabalhos/inic/pdf/IC6-8.pdf>.

Fazard, A. \& Gulliver, T. A. (2019). Log message anomaly detection and classification using Auto-B/LSTM and Auto-GRU. ResearchGate.

Gazzola, M. G. (2017). Um método para avaliação automática da qualidade de recursos educacionais abertos usando deep learning. < http://www.brie.org/pub/index.php/sbie/article/view/7678/5473>.

Graves A. \& Jaitly, N. (2014). Towards end-to-end speech recognition with recurrent neural networks. < http://proceedings.mlr.press/v32/graves14.pdf>.

Graves, A. \& Schmidhuber, J. (2009). Offline handwriting recognition with multidimensional recurrent neural networks. Advances in Neural Information Processing Systems, 545-552.

Haykin, S. (2005). Neural networks: a comprehensive foundation. New Delhi: Pearson Prentice Hall.

Lawal, A. (2021). Wind speed prediction using hybrid 1D CNN and BLSTM network. IEEE Acess, 9(1). https://doi.org/10.1109/ACCESS.2021.3129883.

Lopes, L. P. (2018). Predição do preço do café naturais brasileiro por meio de modelos de statistical machine learning. Sigmae, 7(1), 1-16.

Lu, W., Li, J., Li, Y., Sun, A. \& Wang, J. (2020). A CNN-LSTM-based model to forecast stock prices. Complexity. https://doi.org/10.1155/2020/6622927.

Martins, L. S. F. (2021). Análise da previsão do cenário de produção e cultivo do café arábica no Brasil. Trabalho de Conclusão de Curso. Medianeira, PR, UTFPR.

Marujo, L. (2021). Estudo comparativo entre métodos estatísticos e de inteligência artificial para previsão do preço do café no Brasil. Dissertação de mestrado. Medianeira, PR, UTFPR.

Mousa, A. E. \& Schuller, B. (2016). Deep bidirectional long short-term memory recurrent neural etworks for grapheme-to-phoneme conversion utilizing complex many-to-many alignments. In: Interspeech 2016, San Francisco, USA.

Nelson, M. Q., Pereira, A C. M. \& Oliveira R. A. (2017). Stock market's price prediction with LSTM neural networks. In: International Joint Conference of Neural Networks (IJCNN), Anchorage, Alaska.

Novanda R. R. et al. (2018). A comparison of various forecasting techniques for coffee prices. https://iopscience.iop.org/article/10.1088/1742$6596 / 1114 / 1 / 012119 /$ pdf.

Pacheco, A. G. C. (2016). Classificação de espécies de peixe utilizando redes neurais convolucional. https://arxiv.org/pdf/1905.03642.pdf.

Pinheiro, T. C., Santos, J. A. A. \& Pasa, L. A. (2020). Gestão da produção de frangos de corte por meio de redes neurais. Revista Holos, 2(1), 1-15. https://doi.org/10.15628/holos.2020.9043.

Santos, J. A. A. (2021). Aplicação de redes neurais artificiais na previsão do preço do milho no estado do Paraná. Revista Engenharia e Tecnologia, 13(2), 124-134.

Santos, J. A. A. \& Chaucoski, Y. (2020). Previsão do consumo de energia elétrica na região sudeste: um estudo de caso usando SARIMA e LSTM. Revista CEREUS, 12(1). https://doi.org/10.18605/2175-7275/cereus.v12n4p93-104.

Santos, J. A. A. \& Spancerski, J. S. (2021). Previsão da produtividade de arroz: uma aplicação de redes neurais recorrentes LSTM. Revista CEREUS, 13(2), 163-175. https://doi.org/10.18605/2175-7275/cereus.v13n2p163-175.

Silva, C. A. G. (2018). Previsão do preço da commodity café arábica: Uma aplicação da Metodologia Box-Jenkins. Revista Espacios, 30(4).

SINDICAFÉ-MG (2022). O café no mundo. http://sindicafe-mg.com.br/cafe-no-mundo. 
Research, Society and Development, v. 11, n. 3, e3511326101, 2022

(CC BY 4.0) | ISSN 2525-3409 | DOI: http://dx.doi.org/10.33448/rsd-v11i3.26101

Sun, Q., Jankovic, M. V. \& Bally, L. (2018). Predicting blood glucose with an LSTM and Bi-LSTM based deep neural network. https://arxiv.org/abs/1809.03817. Acesso em: 25 set. 2021.

Tatagiba, S. D., Pezzopane, J. E. \& Reis, E. F. (2010). Crescimento vegetativo de mudas de café arábica submetidas a diferentes níveis de sombreamento. Coffee Science, 5(3): 251-261.

Zao, Z. (2017). LSTM network: a deep learning approach for short-term traffic forecast. IET Intelligent Transport Systems. 11(2), 68-75. https://doi.org/10.1049/iet-its.2016.0208. 